\title{
Estimation of LDL Particle Size Using Lipid Indices: A Population-Based Study of 1578 Schoolchildren
}

\author{
Yaeko Watabe, MD, Osamu Arisaka, MD, PhD, ${ }^{1}$ Noriko Miyake, MD, PhD, ${ }^{2}$ Go Ichikawa, MD, PhD, \\ Satomi Koyama, MD, PhD, and Naoto Shimura, MD, $\mathrm{PhD}^{1}$
}

\begin{abstract}
Background: Low-density lipoprotein (LDL) is atherogenic and LDL particles are reduced in diameter in the presence of insulin resistance, forming small, dense LDL. This study was conducted to assess the relationship between commonly used lipid indices and LDL particle size and furthermore to clarify the best surrogate lipid markers that could conveniently be used to estimate LDL particle size in children.

Methods: We determined LDL particle diameter by gradient gel electrophoresis in 1578 children aged 10-12 years. At the fasting state, the relationships between measured LDL particle size and lipid variables [total cholesterol (TC), triglycerides (TG), LDL-cholesterol (LDL-C), high-density lipoprotein cholesterol (HDL-C), atherogenic index [(TC-HDL-C)/HDL-C, TG/HDL-C, LDL-C/HDL-C, and LDL-C/apolipoprotein B (Apo B) and non-HDL-C (TC-HDL-C)] were analyzed.

Results: The LDL particle diameter was 26.64 (mean) \pm 0.48 (SD) $\mathrm{nm}$ in boys $(n=820)$ and $26.66 \pm 0.49 \mathrm{~nm}$ in girls $(n=758)$; there was not a statistically significant difference. There were statistically significant correlations between LDL particle size and TG or HDL-C concentrations $(r=0.28 \sim 0.37)$, but the correlations with LDL-C and ApoB were very weak. The combined lipid measures, such as atherogenic index, TC/HDL-C, TG/HDL-C, and LDL-C/HDL-C showed moderate correlations $(r=0.33 \sim 0.38)$ with LDL particle size; however, the correlation of non-HDL-C with LDL particle size was weak $(r=0.18 \sim 0.19)$. Simple HDL-C measure appeared to be of comparable value to combined lipid measures.

Conclusions: Our data indicate that various lipid indices are not superior to HDL-C levels alone as a clinical tool for estimating LDL particle size. Non-HDL-C was less valuable in this aspect.
\end{abstract}

\section{Introduction}

$\mathrm{L}$ OW-DENSITY LIPOPROTEIN (LDL) particles are heterogeneous in density, size, lipid composition, and pathogenetic properties. ${ }^{1-3}$ The smaller and denser they are, the stronger their atherogenic impact, possibly because of the low binding affinity of small, dense LDL particles to the LDL receptor, their low resistance to oxidative stress, their prolonged half-life in plasma, and their easy penetration of the arterial intima due to small particle size. ${ }^{4,5}$ It has been demonstrated in many studies that predominance of small dense LDL (sdLDL; LDL particle size of $\leq 25.5 \mathrm{~nm}$ ) is associated with increased risk of coronary artery disease and the presence of sdLDL is a predictor of cardiovascular events. ${ }^{6,7}$ As cardiovascular risk factors persist over time from childhood to adulthood, ${ }^{8,9}$ the presence of sdLDL in childhood is predictive of coronary heart disease in adulthood. In addition, since smaller LDL particle diameter is associated with increases in insulin resistance, ${ }^{10-13}$ detecting sdLDL in the plasma is recognized as a useful tool for the prediction and screening of lifestyle-related diseases that appear to be associated with insulin resistance. ${ }^{14,15} \mathrm{It}$ has been reported that higher fasting insulin level was also correlated with smaller mean LDL particle size in boys. ${ }^{16}$ However, data on the distribution of LDL particle size and its association with cardiovascular risk factors are limited in children.

The gold standard for assessing LDL particle size is gradient electrophoresis, a sophisticated technique that cannot be used on a regular basis in clinical laboratories. ${ }^{1,2}$ Therefore, the measurement of LDL particle diameter is not routinely performed in the clinical setting. As surrogate markers of LDL particle size, the relationships between LDL particle size and several lipid indices easily derived

\footnotetext{
${ }^{1}$ Department of Pediatrics, Dokkyo Medical University, Mibu, Kitakobayashi, Japan.

${ }^{2}$ Department of Clinical Pathology, Juntendo Nerima Hospital, Tokyo, Japan.
}

(C) Yaeko Watabe, et al., 2016: Published by Mary Ann Liebert, Inc. This Open Access article is distributed under the terms of the Creative Commons Attribution Noncommercial License (http://creativecommons.org/licenses/by-nc/4.0/) which permits any noncommercial use, distribution, and reproduction in any medium, provided the original author(s) and the source are credited. 
from a lipid panel, such as LDL-C/apolipoprotein B (Apo $\mathrm{B}),{ }^{17}$ atherogenic index, ${ }^{18}$ total cholesterol (TC)/highdensity lipoprotein cholesterol (HDL-C), ${ }^{19}$ triglycerides (TG)/HDL-C, ${ }^{20}$ and non-HDL-C, ${ }^{21}$ have been proposed in adults with inconsistent results; however, investigations in children have been limited.

Therefore, the objectives of this study were to investigate the relationship between measured LDL particle size and various lipid parameters in a large population-based sample of children, and to then clarify the best surrogate markers to estimate LDL particle size.

\section{Methods}

\section{Subjects}

In total 1,578 children ( 820 boys and 758 girls), fourth grade elementary school students (10 years) and first year junior high school students (12 years), participated in this school-based health survey of lifestyle-related disease, conducted between 2008 and 2012 in Fujioka town in the province of Tochigi, Japan. The population of this town is 18,000 , with one-half of the people working as farmers and one-half commuting to nearby large cities. The town has four elementary schools and two junior high schools.

The examinations were carried out in September-October at the school. In order to obtain overnight fast blood samples, students were allowed to bring a packet box breakfast, which they took after the health examination at the schoolroom.

Written informed consent was obtained from the guardian for the physical examination. The study was approved by the ethics committee of Dokkyo Medical University.

\section{Measurements of lipids and blood pressure}

Fasting blood sampling and standardized measurement of systolic blood pressure and diastolic blood pressure were performed at school. Blood pressure was measured in the sitting position after a 5 min rest by trained observers with an electronic sphygmomanometer (H- 55,TERUMO) using a cuff that was appropriate to the size of the child's upper right arm.

Blood samples collected in collection tubes containing $1 \mathrm{~g} / \mathrm{L}$ EDTA were sent within $3 \mathrm{~h}$ to the laboratory. Plasma was separated and stored at $-80^{\circ} \mathrm{C}$ until measurement of LDL particle size.

Levels of TC (Cholestest CHO, Daiichi Pure Chemicals Co. Ltd.) and TG (Aqua-auto TG-II, Kainos Laboratories, Inc.) were determined using enzymatic methods. HDL-C was measured by precipitation of other lipoproteins with the direct method (Cholestest N HD, Daiichi Pure Chemicals Co. Ltd.). Low-density lipoprotein cholesterol (LDL-C) was calculated by using the Friedewald formula. Apolipoprotein $\mathrm{B}$ (ApoB) was quantified by turbidimetric immunoassay (ApoB auto N, Daiichi Pure Chemicals Co. Ltd.). Among the total cohort of 1578 children, 381 boys and 353 girls underwent measurement of Apo B due to insufficient amount of blood sampling. The atherogenic index (AI) was calculated as follows: $\mathrm{AI}=[\mathrm{TC}-\mathrm{HDL}-\mathrm{C}] / \mathrm{HDL}-\mathrm{C}{ }^{22}$

\section{Determination of $L D L$ particle size}

The LDL particle diameter was determined according to the method described by Krauss et al., ${ }^{1}$ by gradient gel electrophoresis on $2.5 \%$ to $16 \%$ polyacrylamide. After the gels were equilibrated at $120 \mathrm{~V}$ for $20 \mathrm{~min}$, electrophoresis was performed for each gel containing the serum sample diluted 1:2 with sample buffer (comprised of $31 \%$ sucrose, $0.06 \%$ EDTA-2Na, and $0.01 \%$ BPP) to a volume of $20 \mathrm{~mL}$. Each gel also contained thyroglobulin, apoferritin, and latex beads as reference standards of known diameter. The gels were then electrophoresed at $120 \mathrm{~V}$ for $19 \mathrm{~h}$ and then stained for lipid with Oil Red $\mathrm{O}$ that was heated to $55^{\circ} \mathrm{C}$ for $24 \mathrm{~h}$, and for protein with Coomassie Brilliant Blue for $15 \mathrm{~min}$. They were then destained with ethanol and immersion in acetic acid. The gels were then scanned with an image scanner (Epson GT-6500: Seiko Epson Corporation) and analyzed using an image processing and analysis program for Macintosh (National Institutes of Health Image 1.61). Migration distances were determined. The LDL particle diameter was then calculated by comparing the mobility of the sample with the mobility of three calibrated standards on each gel. Small dense LDL was defined as LDL with a particle diameter $\leq 25.5 \mathrm{~nm}$, based on the criteria proposed by Austin et al. ${ }^{23}$

\section{Statistical analysis}

All statistical analyses were performed using SPSS 15.0 for Windows. Unpaired Student's $t$-test was used to compare various parameters between the groups. The relationships between LDL particle size and variables were analyzed using parametric tests; relationships between the variables are reported as Pearson correlation coefficients. A $P$ value $<0.05$ was considered statistically significant.

\section{Results}

The baseline characteristics of the study population are shown in Table 1.

Table 1. Background Anthropometric, Blood Pressure, and Lipid Data of 1578 Schoolchildren

\begin{tabular}{lccc}
\hline & Boys $(\mathrm{n}=820)$ & Girls $(\mathrm{n}=758)$ & P value \\
\hline Body mass index (Z-score) & $0.1 \pm 1.1$ & $-0.1 \pm 0.9$ & $<0.05$ \\
Systolic blood pressure (mmHg) & $105.1 \pm 10.9$ & $104.0 \pm 10.9$ & $<0.05$ \\
Diastolic blood pressure (mmHg) & $57.9 \pm 8.6$ & $58.5 \pm 8.4$ & n.s. \\
TC (mg/dL) & $168.1 \pm 27.0$ & $171.4 \pm 25.6$ & $<0.05$ \\
LDL-C (mg/dL) & $92.4 \pm 23.5$ & $95.6 \pm 22.2$ & $<.005$ \\
HDL-C (mg/dL) & $68.1 \pm 14.1$ & $67.2 \pm 12.7$ & n.s. \\
TG (mg/dL) & $58.9 \pm 30.8$ & $63.2 \pm 30.1$ & $<0.01$ \\
Apo B (mg/dL) & $69.6 \pm 19.8(n=381)$ & $72.6 \pm 19.4(n=353)$ & $<0.05$ \\
\hline
\end{tabular}

Values are expressed as mean \pm standard deviation.

Apo B, apolipoprotein B; HDL-C, high-density lipoprotein cholesterol, LDL-C, low-density lipoprotein cholesterol; TG, triglycerides. 
Table 2. Correlation of LDL Particle Size and Body Mass Index, Blood Pressures, LIPIDS, AND VARIOUS LIPID INDICES

\begin{tabular}{|c|c|c|c|c|}
\hline & \multicolumn{2}{|c|}{ Boys $(\mathrm{n}=820)$} & \multicolumn{2}{|c|}{ Girls $(\mathrm{n}=758)$} \\
\hline & $\mathrm{r}$ & $\mathrm{P}$ value & $\mathrm{r}$ & $\mathrm{P}$ value \\
\hline Body mass index (Z-score) & -0.15 & $<0.0001$ & -0.08 & $<0.05$ \\
\hline Systolic blood pressure & -0.001 & n.s. & -0.01 & n.s. \\
\hline Diastolic blood pressure & -0.01 & n.s. & -0.03 & n.s. \\
\hline TC & 0.02 & n.s. & 0.01 & n.s. \\
\hline LDL-C & -0.12 & $<0.0001$ & -0.13 & $<0.0001$ \\
\hline HDL-C & 0.37 & $<0.0001$ & 0.37 & $<0.0001$ \\
\hline TG & -0.31 & $<0.0001$ & -0.28 & $<0.0001$ \\
\hline ApoB & $-0.13(n=381)$ & $<0.01$ & $-0.06(n=353)$ & n.s. \\
\hline Atherogenic index & -0.35 & $<0.0001$ & -0.38 & $<0.0001$ \\
\hline TC/HDL-C & -0.35 & $<0.0001$ & -0.38 & $<0.0001$ \\
\hline TG/HDL-C & -0.37 & $<0.0001$ & -0.36 & $<0.0001$ \\
\hline LDL-C/HDL-C & -0.33 & $<0.0001$ & -0.35 & $<0.0001$ \\
\hline non-HDL-C & -0.18 & $<0.0001$ & -0.19 & $<0.0001$ \\
\hline LDL-C/ApoB & $-0.03(n=381)$ & n.s. & $-0.08(n=353)$ & n.s. \\
\hline
\end{tabular}

n.s., not significant; TC, total cholesterol.

1. The LDL particle diameter was 26.64 (mean) \pm 0.48 (Standard deviation) $\mathrm{nm}$ in boys $(n=820)$ and $26.66 \pm 0.49 \mathrm{~nm}$ in girls $(n=758)$, which was not significantly different.

2. The prevalence of small, dense LDL (LDL with a particle diameter $\leq 25.5 \mathrm{~nm})$ in was $0.6 \%(5 / 820)$ in boys and $2 \%$ in girls $(15 / 758)$.

3. Correlations coefficients of the measured LDL particle diameter versus cardiovascular risk factors (i.e., lipids, blood pressure, and body mass index) are shown in Table 2. Varying degrees of statistically significant correlations between LDL particle size and cardiovascular risk factors were observed except for serum TC and blood pressure.

4. Correlations coefficients of measured LDL particle diameter versus surrogate markers [i.e., atherogenic index (AI) [(TC-HDL-C)/HDL-C], TC/HDL-C, TG/ HDL-C, HDL-C/LDL-C, and LDL-C/Apo B, and nonHDL-C)] for estimating LDL particle diameter are shown in Table 2. The correlation between LDL particle size and AI is shown in Fig. 1.

\section{Discussion}

To date, limited information is available on the distribution of LDL particle size in large population-based samples of children and adolescents. This study used strict fasting blood samples collected from more than 1500 subjects, thus eliminating the effects of postprandial elevation of TG or insulin on lipoprotein metabolism and LDL particle size. ${ }^{4,18}$

In terms of LDL particle size by sex, despite methodological differences, higher mean LDL particle sizes have been reported in females compared with males. ${ }^{3,24} \mathrm{We}$ did not observe any such difference by sex in our study [26.64 nm (boys) vs. $26.66 \mathrm{~nm}$ (girls)]. Further investigations are needed to confirm sexual differences in children and adolescents.

The prevalence of the sdLDL phenotype was only $1.3 \%$ in our cohort and was similar between sexes and across ages. Shimabukuro et al. ${ }^{3}$ reported a prevalence of $10.8 \%$ in prepubertal Japanese boys and $4.4 \%$ in prepubertal Japanese girls by the same gradient gel electrophoresis method. Freedman et al. ${ }^{25}$ reported that the prevalence of the small, dense LDL phenotype ranged from 5\% in black boys and girls to $13 \%$ in white boys, using nuclear magnetic resonance spectroscopy. These different observations may be caused by varying effects of genetic and environmental factors affecting LDL particle size. ${ }^{26-28}$

With regard to correlations between LDL particle size and simple lipid measures, moderate correlations were observed between LDL particle size and both TG and HDL-C concentrations; correlation coefficients by sex were -0.31 (boys) and -0.28 (girls) and 0.37 (both sex), respectively. In contrast, the correlations between LDL particle size and LDL-C and ApoB were weak but statistically significant.

Metabolically, hypertriglyceridemia promotes TG transfer from very low-density lipoprotein (VLDL) to HDL. The TG-enriched HDL then transfers TG to LDL and removes cholesterol from LDL; thus, the cholesterol-depleted LDL

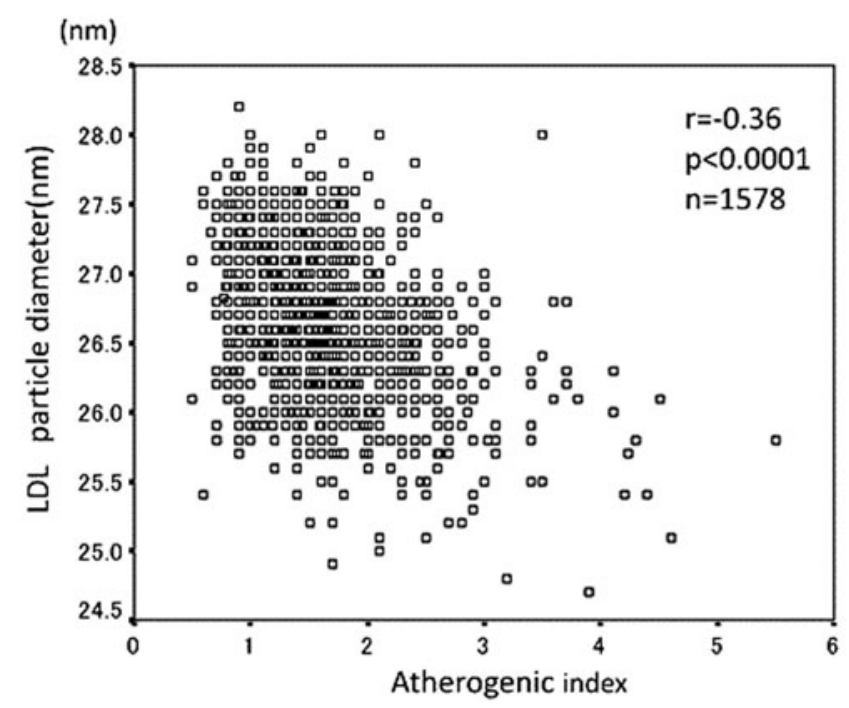

FIG. 1. The correlation between low-density lipoprotein (LDL) particle size and atherogenic index in both sexes. 
then becomes smaller and denser. In the presence of hypertriglyceridemia, the cholesteryl ester transfer protein allows cholesteryl esters to be transferred from LDL in exchange for a TG molecule from VLDL. TG is then hydrolyzed by hepatic triglyceride lipase or lipoprotein lipase to produce smaller, denser LDL particles. ${ }^{2-4,18}$ The activities of cholesteryl ester transfer protein and hepatic triglyceride lipase are enhanced by increased adiposity or increased insulin resistance. A reduction in LDL particle size associated with a decrease in the HDL-C level may result from the alteration in lipoprotein metabolism caused by insulin resistance. ${ }^{10,13,29}$ Thus, TG and HDL-C concentrations are considered to correlate well with LDL particle size.

In our investigation the correlation between Apo B and LDL particle size was poor, but ApoB can be considered as a surrogate measurement of LDL particle number, because each LDL particle carries one apolipoprotein B-100 molecule. Assessment of abnormalities in LDL particle number in addition to LDL particle size is important for qualitative evaluation of the atherogenicity caused by LDL particles. ${ }^{15,30}$

With regard to the correlations between LDL particle size and combined lipid measures, lipid ratios that include information on at least two measures might provide a more integrated and concentrated explanation than single lipid measures; even TG or HDL-C appeared to exhibit pretty good correlations with LDL particle size. Therefore, several lipid ratios have been proposed as convenient clinical indicators because of the integrative information provided by multiple variables. ${ }^{19,31,32}$ In our observations the same degree of moderate correlations (i.e., correlation coefficients: $-0.33 \sim-0.38$ ) between LDL particle size and the AI, TC/ HDL-C, TG/HDL-C, and LDL-C/HDL-C were found. However, the correlations between LDL particle size and non-HDL-C, LDL/Apo B were weaker. Therefore, it appeared that simple HDL-C measurement had similar value to AI, TC/HDL-C, TG/HDL-C, and LDL-C/HDL-C.

Among these parameters, the TG/HDL-C ratio has been reported as an indicator of insulin resistance ${ }^{33-35}$ and the AI as a predictive value for cardiovascular disease. ${ }^{22,36} \mathrm{Re}-$ cently, the TG/HDL-C ratio has been reported to have a good correlation with homeostasis model assessment for insulin resistance, and as a marker of insulin resistance that is an essential metabolic background of reduction of LDL particle size. ${ }^{37-39}$ However, in the present study TG/HDL-C appeared to not be superior to other lipid indices. It was considered that any lipid indices (combining lipid measures, including either TG or HDL-C) seemed to correlate with LDL particle size to the same degree. Similar observations have been reported in adults. ${ }^{40}$

Recently, it has been reported that non-HDL-C, calculated as the difference between TC and HDL-C levels, is well associated with metabolic syndrome in youths, and screening for metabolic syndrome based on non-HDL-C concentration is thought to have an advantage in the pediatric setting because pretest fasting is not required. ${ }^{21,41}$ However, contrary to our expectation, the correlation between non-HDL-C and LDL particle size was the weakest among the lipid parameters investigated. A similar observation has been reported elsewhere. ${ }^{42,43}$ Although the underlying mechanisms linking non-HDL-C and pediatric metabolic syndrome remain to be elucidated, our data indicate that non-HDL-C is not a good tool for the prediction of metabolic syndrome.
In conclusion, our data indicate that various lipid indices are not superior to HDL-C levels alone as a clinical tool for estimating LDL particle size. As surrogate markers to predict LDL particle size, lipid indices (combined lipid measures), including TG or HDL-C had similar value, but non-HDL-C was less valuable.

\section{Acknowledgments}

This study was supported by grants from the Ministry of Health, Labour, and Welfare of Japan, in the areas of the establishment of pathophysiology, diagnosis, and management of metabolic syndrome in childhood and early management of obesity in childhood. We thank Mrs. Yasuyo Kawai for her excellent technical assistance.

Y. Watabe analyzed and interpreted the data, generated the figures, and drafted the initial manuscript; G. Ichikawa, S. Koyama, and N. Shimura collected data and reviewed the manuscript; N. Miyake analyzed and interpreted the data and reviewed the manuscript; O. Arisaka conceived and designed the study and reviewed and revised the manuscript. All authors approved the final manuscript as submitted.

\section{Author Disclosure Statement}

No competing financial interests exist.

\section{References}

1. Krauss RM. Heterogeneity of plasma low-density lipoproteins and atherosclerosis risk. Curr Opin Lipidol 1994;5: 339-349.

2. Arisaka O, Fujiwara S, Yabuta K, et al. Characterization of low-density lipoprotein subclasses in children. Metabolism 1997;46:146-148.

3. Shimabukuro T, Sunagawa M, Ohta T. Low-density lipoprotein particle size and it regulatory factors in school children. J Clin Endocrinol Metab 2004;89:2923-2927.

4. Mikhailidis DP, Elisaf M, Rizzo M, et al. European panel on low density lipoprotein (LDL) subclasses: A statement on the pathophysiology, atherogenicity and clinical significance of LDL subclasses: Executive summary. Curr Vasc Pharmacol 2011;9:531-532.

5. Chapman MJ, Guérin M, Bruckert E. Atherogenic, dense low-density lipoproteins. Pathophysiology and new therapeutic approaches. Eur Heart J 1998;19:A24-30.

6. Rizzo M, Pernice V, Frasheri A, et al. Small, dense lowdensity lipoproteins (LDL) are predictors of cardio- and cerebro-vascular events in subjects with the metabolic syndrome. Clin Endocrinol 2009;70:870-875.

7. Nishikura T, Koba S, Yokota Y, et al. Elevated small dense low-density lipoprotein cholesterol as a predictor for future cardiovascular events in patients with stable coronary artery disease. J Atheroscler Thromb 2014;21:755-767.

8. Juhola J, Magnussen CG, Viikari JS, et al. Tracking of serum lipid levels, blood pressure, and body mass index from childhood to adulthood: The Cardiovascular Risk in Young Finns Study. J Pediatr 2011;159: 584-590.

9. Kaitosaari T, Simell O, Viikari J, et al. Tracking and determinants of LDL particle size in healthy children from 7 to 11 years of age: The STRIP Study. Eur J Pediatr 2009; 168:531-539.

10. Reaven GM, Chen YD, Jeppesen J, et al. Insulin resistance and hyperinsulinemia in individuals with small, dense, lowdensity lipoprotein particles. J Clin Invest 1993;92:141-146. 
11. Haffner SM, Mykkanen L, Robbins D, et al. A preponderance of small dense LDL is associated with specific insulin, proinsulin and the components of the insulin resistance syndrome in non- diabetic subjects. Diabetologia 1995;38:1328-1336.

12. Grundy SM. Small LDL, atherogenic dyslipidemia, and the metabolic syndrome. Circulation 1997;95:1-4

13. Toth PP. Insulin resistance, small LDL particles, and risk atherosclerotic disease. Curr Vasc Pharmacol 2014;12: 653-657.

14. Taşcılar ME, Özgen T, Cihan M, et al. The effect of insulin resistance and obesity on low-density lipoprotein particle size in children. J Clin Res Pediatr Endocrinol 2010;2:63-66.

15. Burns SF, Lee SJ, Arslanian SA. Surrogate lipid markers for smal dense low- density lipoprotein particles in overweight youth. J Pediatr 2012;161:991-996.

16. Shea S, Aymong E, Zybert P, et al. Fasting plasma insulin modulates lipid levels and particle sizes in 2- to 3-year-old children. Obes Res 2003,11:709-721.

17. Wägner AM, Jorba O, Rigla M, et al. LDL-cholesterol/ apolipoprotein B ratio is a good predictor of LDL phenotype B in type 2 diabetes. Acta Diabetol 2002;39:215-220.

18. Kojima M, Kanno H, Yamazaki Y, et al. Association of low-density lipoprotein particle size distribution and cardiovascular risk factors in children. Acta Paediatr 2005; 94:281-286.

19. Maruyama C, Imamura K, Teramoto T. Assessment of LDL particle size by triglyceride / HDL-cholesterol ratio in non-diabetic, healthy subjects without prominent hyperlipidemia. J Atheroscler Thromb 2003;10:186-191.

20. Yoshida A, Kouwaki M, Matsutani Y, et al. Usefulness of serum total cholesterol/triglyceride ratio for predicting the presence of small, dense LDL. J Atheroscler Thromb 2004; 11:215-219.

21. Belani SS, Goldberg AC, Coyne DW. Ability of non-highdensity lipoprotein cholesterol and calculated intermediatedensity lipoprotein to identify nontraditional lipoprotein subclass risk factors in dialysis patients. Am J Kidney Dis 2004;43:320-329.

22. Castelli WP, Abbott RD, McNamara PM. Summary estimates of cholesterol used to predict coronary heart disease. Circulation 1983;67:730-734.

23. Austin MA, Breslow JL, Hennekens CH, et al. Low-density lipoprotein subclass patterns and risk of myocardial infarction. JAMA 1988;260:1917-1921.

24. Freedman DS, Bowman BA, Otvos JD, et al. Levels and correlates of LDL and VLDL particle sizes among children: The Bogalusa heart study. Atherosclerosis 2000;152:441-449.

25. Freedman DS, Otvos JD, Jeyarajah EJ, et al. Sex and age differences in lipoprotein subclasses measured by nuclear magnetic resonance spectroscopy: The Framingham Study. Clin Chem 2004;50:1189-1200.

26. Austin MA.Genetic and environmental influences on LDL subclass phenotypes. Clin Genet 1994;46:64-70.

27. Bossé Y, Pérusse L, Vohl MC. Genetics of LDL particle heterogeneity: From genetic epidemiology to DNA-based variations. J Lipid Res 2004;45:1008-1026.

28. Ryder JR, Vega-López S, Ortega R, et al. Lifestyle intervention improves lipoprotein particle size and distribution without weight loss in obese Latino adolescents. Pediatr Obes 2013;8:e59-63.

29. Hayashibe H, Asayama K, Nakane T, et al. Increased plasma cholesteryl ester transfer activity in obese children. Atherosclerosis 1997;129:53-58.
30. Arisaka O, Ichikawa G, Koyama S, et al. Relation between low-density lipoprotein particle size and insulin and diabetes mellitus. J Pediatr 2009;155:600.

31. Stan S, Levy E, Delvin EE, et al. Distribution of LDL particle size in a population-based sample of children and adolescents and relationship with other cardiovascular risk factors. Clin Chem 2005;51:1192-1200.

32. Décary S, Dumont G, Lamarche B, et al. Assessment of the validity of the frequently used lipid indices for predicting LDL peak particle diameter in a large cohort of 1955 normal and dyslipidemic subjects. Clin Biochem 2010;43: 401-406.

33. Srisawasdi P, Chaloeysup S, Teerajetgul Y, et al. Estimation of plasma small dense LDL cholesterol from classic lipid measures. Am J Clin Pathol 2011;136:20-29.

34. McLaughlin T, Abbasi F, Cheal K, et al. Use of metabolic markers to identify overweight individuals who are insulin resistant. Ann Intern Med 2003;139:802-809.

35. McLaughlin T, Reaven G, Abbasi F, et al. Is there a simple way to identify insulin-resistant individuals at increased risk of cardiovascular disease? Am J Cardiol 2005;96:399_ 404.

36. Hannon TS, Bacha F, Lee SJ, et al. Use of markers of dyslipidemia to identify overweight youth with insulin resistance. Pediatr Diabetes 2006;7:260-266.

37. Yang RL, Shi YH, Hao G, et al. Increasing oxidative stress with progressive hyperlipidemia in human: Relation between malondialdehyde and atherogenic index. $J$ Clin Biochem Nutr 2008;43:154-158.

38. Chiang JK, Lai NS, Chang JK, et al. Predicting insulin resistance using the triglyceride-to-high-density lipoprotein cholesterol ratio in Taiwanese adults. Cardiovasc Diabetol 2011;10:93.

39. Kang HT, Yoon JH, Kim JY, et al. The association between the ratio of triglyceride to HDL-C and insulin resistance according to waist circumference in a rural Korean population.Nutr Metab Cardiovasc 2012;22:1054-1060.

40. St-Pierre AC, Cantin B, Dagenais GR, et al. The triglyceride/high-density lipoprotein cholesterol ratio, the small dense low-density lipoprotein phenotype, and ischemic heart disease risk. Metab Syndr Relat Disord 2004;2:57-64.

41. Li C, Ford ES, McBride PE, et al. Non-high-density lipoprotein cholesterol concentration is associated with the metabolic syndrome among US youth aged $12-19$ years. $J$ Pediatr 2011;158:201-207.

42. Kimm H, Lee SW, Lee HS, et al. Associations between lipid measures and metabolic syndrome, insulin resistance and adiponectin. Usefulness of lipid ratios in Korean men and women. Circ J 2010;74:931-937.

43. Kim SW, Jee JH, Kim HJ, et al. Non-HDL-cholesterol/ HDL-cholesterol is a better predictor of metabolic syndrome and insulin resistance than apolipoprotein B/apolipoprotein A1. Int J Cardiol 2013;168 (3):2678-83.

Address correspondence to: Osamu Arisaka, $M D, P h D$

Department of Pediatrics

Dokkyo Medical University

880 Kitakobayashi, Mibu, Tochigi 321-0293

Japan

E-mail: arisaka@dokkyomed.ac.jp 